oesophagitis delayed treatment in $12.1 \%$ and hiatus hernia repair surgery was required in $1.3 \%$ of patients. Stricture rates were 5.4\% requiring a median of 2 (IQR:6) dilatations and bleeding requiring hospital admission was $0.45 \%$. RFA failed or was abandoned in $6.3 \%$ of patients. Metachronous lesions development during RFA treatment phase warranting further therapy in $14.9 \%$ patients: BO (2.7\%) LGD (0.9\%) HGD (5\%) and IMC (6.3\%). Metachronous lesions and BO developed after CRIM in 5\% requiring further therapy and $0.9 \%$ developed invasive cancer. Median follow up post CRD/CRIM was $20.1( \pm 28.9)$ months. The survival rate over the 10 -year study period was $94.6 \%$ with the majority of deaths due to unrelated disease.

Conclusions This 10-year data demonstrates that RFA therapy is effective in achieving eradication of $\mathrm{BO}$ and dysplasia with a favourable safety profile.

\section{PTU-059 EMR FOR EARLY BARRETT'S NEOPLASIA: 15-YEARS OF EXPERIENCE FROM A UK TERTIARY REFERRAL CENTRE}

\begin{abstract}
${ }^{1,2} \mathrm{JR}$ White* ${ }^{*}{ }^{1,2} \mathrm{~J}$ Ortiz-Fernández-Sordo, 1,2 J Santiago-García, 1,2 D Reddiar, 1,3 J De Caestecker, ${ }^{1,4} \mathrm{~A}$ Cole, ${ }^{1,2} \mathrm{P}$ Kaye, ${ }^{1,2} \mathrm{~K}$ Ragunath. ${ }^{1}$ Nottingham Digestive Diseases Centre, The University of Nottingham, UK; ${ }^{2}$ NIHR BRC at Nottingham University Hospitals and University of Nottingham, UK; ${ }^{3}$ Leicester General Hospital, UK; ${ }^{4}$ Royal Derby Hospital, UK
\end{abstract}

\subsection{6/gutjnl-2019-BSGAbstracts.272}

Introduction Endoscopic mucosal resection (EMR) is a widely used therapy for visible dysplastic lesions associated with Barrett's oesophagus (BO) and intramucosal adenocarcinoma (IMC). This study evaluates the efficacy and safety of a single high-volume UK tertiary centre with 15 -years experience.

Methods A retrospective review was conducted of patients referred to Nottingham University Hospital between 20042019 for EMR with dysplastic BO visible lesions or IMC. The main outcomes were endoscopic resection success rates, long-term recurrence rates, complications during the treatment phase, surgery rates, median follow up prior to discharge from tertiary centre and tumour related deaths.

Results A total of 309 lesions were resected in 212 patients, median age was $68.1 \pm 9.4$ years, the male: female ratio was 5:1. Median BO length was C2(IQR:6) M4(IQR6) and 76.2\% of lesions were at the 12 to 6 o'clock position. The most common lesion was Paris IIa (63.4\%) and the median size was $10 \mathrm{~mm}$ (3-70). Most procedures were done under intravenous sedation as a day-case with the ligate and cut technique (93.2\%) and the Duette ${ }^{\circledR}$ multi-band mucosectomy device (88\%). APC was used in addition to EMR in 5.4\% of cases. Complete resection rates were 95.5\%. Prophylactic measures to prevent bleeding were undertaken in $11.3 \%$. Significant complications requiring admission and further treatment was $3.8 \%$ : bleeding $(2.3 \%)$ and perforation $(0.3 \%)$ with a median length of stay of 1 day (1-8). Stricture rates were $2.6 \%$ requiring a median of 1 (IQR:1.75) dilatation. The most commonly resected histological grade was IMC (48.1\%), high grade dysplasia (37\%) and low grade dysplasia (6.5\%). The majority of tumours were stage T1a (86.7\%). 22\% of patients with confirmed adenocarcinoma had an indication for surgery and over half of these underwent surgery. Post EMR $72.5 \%$ had additional therapy for the remaining BO. After a median follow up of 32 months (IQR 43.6) metachronous lesions developed in $10.7 \%$ of patients. $95 \%$ of these were successfully treated with endoscopic or surgical therapy. The survival rate over the study period was $85.7 \%$, with cause of death attributed to unrelated disease $(11.3 \%)$ and oesophageal adenocarcinoma (2.8\%).

Conclusions This real-world data demonstrates that EMR is a minimally invasive, safe and effective treatment for Barrett's neoplasia that can be delivered in a day-case setting. It allows accurate local staging with the option of surgery for locally advanced disease.

\section{Gastroduodenum}

\section{Orals}

\section{OTU-15 INTERMITTENT ADMINISTRATION OF PPI IS NOT INFERIOR TO INFUSION FOLLOWING ACUTE NON- VARICEAL UPPER GASTROINTESTINAL BLEEDING}

${ }^{1}$ Rebecca Smith*, 'Zia Madiah, ${ }^{2}$ Andreea Tanasescu, ${ }^{1}$ Adam Stone. ${ }^{1}$ St Richard's Hospital, Chichester, UK; ${ }^{2}$ QA Hospital, Portsmouth, UK

\subsection{6/gutjnl-2019-BSGAbstracts.273}

Introduction Despite the ubiquitous use of PPI therapy following acute upper gastrointestinal bleeding, the optimal dose and method of administration remains controversial.

Aim To determine whether PPI therapy delivered intermittently was non-inferior to continuous infusion; with regard to rebleeding, mortality, need for surgery and length of hospital stay, in acute upper gastrointestinal bleeding.

Study design Systematic Review and Meta-analysis

Method The MEDLINE database was search with a predefined search strategy. RCTs were considered eligible if they included a group treated with a stat dose of PPI followed by $8 \mathrm{mg} / \mathrm{hr}$ infusion for 72 hours compared to a group treated with an intermittent PPI regimen, following endoscopy for acute upper gastrointestinal bleeding.

Abstracts were screened against eh eligibility criteria. Two reviewers trained in critical appraisal reviewed the full text articles, extracted data and applied the GRADE tool to assess for risk of bias.

Data was synthesised using Mantel-Haenszel fixed-effects method. Heterogenicity was assessed with the $\mathrm{I}^{2}$ statistic and examined as per the study protocol. Non-inferiority margins were pre-defined as $0.962,0.934$ and 0.914 for re-bleeding, need for surgery and mortality respectively. This was determined as $20 \%$ the difference from the odds ratio to the lower bound of a 2-sided 95\% CI taken from infusion versus placebo meta-analysis.

Results 26 RCTs were included $(n=4368)$. Significant heterogeneity was found for the outcome measure length of hospital stay and was not resolved by sub-group analysis or the use of a random-effects model. The risk of rebleeding was lower in the intermittent administration group compared to infusion, although was not statistically significant at either 72 hours (OR 1.03 95\%CI 0.77-1.37) or 30 days (OR 1.05 95\%CI $0.85-1.29)$. This revealed non-inferiority as per the predefined margin. The risk of needing surgery was higher in the intermittent group (OR 0.87 95\%CI 0.63-1.20), whilst this was not statistically significant it did not meet the strict margin set to determine non-inferiority. The risk of mortality also favoured intermittent administration (OR 1.13 95\%CI $0.81-$ 1.58) and showed non-inferiority.

The overall risk of bias was high in 5, undetermined in 6 and low in 15 of the RCTs. 